\title{
Un criterio robusto para la medida del margen -coeficiente- de seguridad
}

\author{
A robust approach for measuring the margin -coefficient- \\ of security
}

1. Cervera $^{(*)}$

RESUMEN

SUMMARY

En este trabajo se analizan en primer lugar algunos inconvenientes de los habituales coeficientes parciales de seguridad — factores multiplicadores de las cargas - como medida del alejamiento a las condiciones de colapso en el caso de las estructuras con anisotropías importantes, como son especialmente aquellas sin resistencia a la tracción -los arcos de fábrica, como ejemplo paradigmático-Para superar dichas dificultades se propone un criterio sencillo y robusto basado en la contracción de la superficie límite, criterio que aporta una medida coincidente con la habitual para los casos isótropos, pero que permite medir igualmente de forma consistente el alejamiento a las condiciones del colapso para el resto de las situaciones. Se aplica finalmente el modelo a la determinación de los márgenes de seguridad en secciones de doble simetría sometidas a compresión esviada para materiales sin, y con resistencia a tracción, así como al problema de la estabilidad de un prisma frente al vuelco, mostrando las mejoras que aporta para caracterizar dichos problemas.

401-11

Palabras Clave: seguridad, colapso plástico, superficie límite, criterios de fallo, arcos de fá-

Keywords: security, plastic collapse, yield surface, failure criteria, masonry arches.

This paper offers a brief analysis of the drawbacks that can be found with the usual partial security coefficients, as loads multipliers, when employed for the measurement of the distance to the collapse condition in such structures, as masonry arches, that have important anisotropies or lacks tensile strength. To deal with such difficulties the paper presents a simple and robust security criterium based on a contraction of the yield surface. That criterium, that performs identically in isotropic structures to those usually employed, can however deal consistently with situations where former criteria fail. The criterium is applied to sections with double symmetry under axial load and unsymmetrical bending, in materials with and without tension resistance. It is also applied to the prism stability problem, showing the advantages in the caracterization of such problems. brica. 


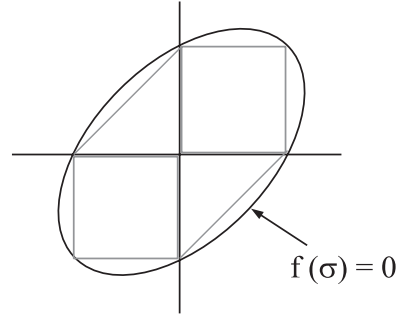

1

1. Criterio de plastificación de Von Mises.

\section{MODELOS DE SEGURIDAD}

Empezaremos con un breve repaso de los modelos de seguridad al uso, para lo que emplearemos como referencia la representación más habitual en los textos de análisis plástico a los estados de esfuerzo, que los identifica como un punto en el espacio cartesiano de las posibles combinaciones entre todos ellos, y en el que los puntos correspondientes a estados de colapso están contenidos en la superficie de fluencia, siendo los puntos interiores puntos de estado admisible para la estructura, y los exteriores puntos que representan combinaciones de esfuerzos inalcanzables. Un ejemplo clásico de dicha representación, para el caso de la plastificación de un punto de un material metálico sometido a estados planos de esfuerzo, y en el que los ejes cartesianos representan las tensiones principales, es el de la Figura 1, correspondiente al criterio de resistencia de Von Mises. Hay muchas otras referencias posibles, aunque una ya clásica en el análisis de las estructuras de fábrica ${ }^{1}$ es (3), como lo es (5) en estructuras de hormigón.

Supondremos en lo sucesivo que la superficie se representa mediante la expresión $f(\sigma)=0$ en la que $\sigma$ representa el vector que define los esfuerzos de comprobación, y supondremos como es usual que puntos interiores a la región delimitada por $f$ son puntos admisibles para valores de $f(\sigma)<0$, mientras que los puntos exteriores corresponden a valores inalcanzables en los esfuerzos, con $f(\sigma)>0$.

En dicha representación, obtener seguridad frente a rotura supone asegurar que cualquiera de los estados de esfuerzo derivados de las diversas condiciones de carga a que la estructura va a estar sometida se sitúe suficientemente alejado de los puntos de colapso representados en dicha superficie, para asegurar que se evita alcanzar alguno de los casos de rotura (Figura 3).

El procedimiento más clásico para establecer un cierto grado de seguridad, asociado a los coeficientes parciales de seguridad, trata de alejarse de las situaciones de rotura considerando tanto reducciones en los valores de las resistencias que representan el comportamiento del material, como ampliaciones en los valores de las cargas respecto de las realmente existentes para establecer las que provocan la rotura en esa estructura de material reducido, mediante un conjunto de factores aplicados a cada una de ellas: la rotura se supone en situaciones de carga mayorada mediante un coeficiente de seguridad que podemos denominar mecánico y cuya determinación y calibración habitual responde a los conocidos como mé- todos probabilísticos de nivel 2 (2). Al objeto de este texto no tiene inicialmente mucha importancia la diferencia de detalle en valores entre los diferentes coeficientes, ni siquiera la distinción entre los dos tipos de coeficiente parcial de seguridad, por lo que podemos imaginar el caso más sencillo para el que la comprobación, en el formato de las normas tradicionales, era del tipo $\sigma_{d} \leq f / \gamma$, formato al que pueden reducirse en los casos lineales las comprobaciones prescritas en la actual normativa, ya sean del tipo:

$$
\sigma_{d}\left(Q \gamma_{f}\right) \leq \frac{f}{\gamma_{m}}
$$

ya sean del más completo:

$$
\psi\left(\frac{E\left(Q \gamma_{f}\right)}{R\left(P / \gamma_{m}\right)}, \ldots\right) \leq 1
$$

donde $\mathrm{E}$ y $\mathrm{R}$ representan respectivamente un esfuerzo efecto de las cargas $Q$ actuantes y la correspondiente resistencia derivada de las propiedades $P$ del material en la configuración estructural empleada, y donde $\psi$ representa el diagrama de interacción que resulte de aplicación para la combinación de esfuerzos considerada, diagrama que es precisamente un caso particular de la superficie de fluencia sin más que escribir²:

$$
f(\boldsymbol{\sigma})=\psi\left(\frac{E\left(Q \gamma_{f}\right)}{R\left(P / \gamma_{m}\right)}, \ldots\right)-1 \leq 0 .
$$

El procedimiento descrito resulta inseguro en situaciones en las que, como en el equilibrio de los arcos, la seguridad puede verse más bien como un problema geométrico. Como aclaración, en la Figura 2 se representa la superficie límite para el plano de una junta rectangular de un arco sin resistencia en tracción, sometido a compresiones excéntricas, y cuyos diagramas de tensiones respondan al modelo rectangular — se representa el estado de tensiones correspondiente a diversos puntos del diagrama de fluencia-.

En el caso señalado de los arcos sin resistencia a tracción, asegurar seguridad frente al colapso consiste habitualmente en asegurar que la resultante de las presiones correspondiente a los distintos estados de carga previstos esté contenida suficientemente dentro de la sección, como medio de asegurar que no se produce la rótula asociada al proceso de colapso. No se trata de un problema de resistencia: el colapso podría producirse aun con material de resistencia infinita en compresión si la sección resulta insuficiente. En este caso lo que se está haciendo implícitamente al acotar la posición de la trayectoria de presiones es configurar un coeficiente de seguridad geométrico que opera en el sentido de reducir la geometría de la sección disponible. La comprobación 
ahora es que la geometría considerada para el cálculo permita el equilibrio, con $g_{d} \leq \mathrm{g} / \gamma$. Puede verse un ejemplo de la aplicación de dicha estrategia en (1). Pero dicha estrategia no resulta suficientemente segura en el problema reseñado en los casos de cargas de pequeña magnitud si no se combina adecuadamente dicho modelo de seguridad con la previsión de hipótesis alternativas de carga, hipótesis en las que se tenga en cuenta la incertidumbre sobre su magnitud y su posición real.

Los casos citados corresponden a situaciones en las que el origen de la gráfica de esfuerzos —o de cargas- está próximo a una de las posibles situaciones de colapso. En casos en los que el origen se sitúa relativamente centrado respecto de las situaciones de colapso, las estrategias anteriores pueden considerarse intercambiables.

Una estrategia alternativa capaz de dar adecuada cuenta de la seguridad en todas las situaciones apuntadas consiste en establecer la seguridad mediante una reducción de la región ocupada por la superficie límite obtenida, no por la vía de la reducción de los esfuerzos máximos, o por la vía de la reducción de la geometría disponible, sino por la vía de la reducción del potencial representado por la función $f(\sigma)$, adoptando por tanto como superficie límite para el cálculo la reducida $f(\sigma)+\gamma=0$, o mediante cualquier procedimiento análogo de contracción de la superficie de fluencia. Esta estrategia debe implicar, para el caso que hemos citado del equilibrio de un arco sin resistencia a tracción, que obtener la seguridad requerida va a exigir la existencia en cualquier caso de un nivel de carga mínimo sin el que la seguridad es inalcanzable. En la Figura 3 vemos representados los tres criterios de seguridad reseñados, y en la Figura 4 podemos observar con claridad la muy diferente forma con que se aborda la corrección de una situación límite partiendo de cada una de estas estrategias.

En la rápida aproximación anterior se han empleado, implícitamente para no distraer en la reflexión, algunos conceptos cuya validez se repasan de forma algo más explícita en el anejo 1, y que resultan esenciales para hacer aceptable la aplicación de las ideas precedentes al caso de las fábricas.

\section{UN MODELO ROBUSTO DE MEDIR EL MARGEN DE SEGURIDAD}

Por lo visto en las anteriores figuras, si el punto de carga nula coincide con el centro de la superficie de rotura, el coeficiente de seguridad no es más que un factor entre dos figuras homólogas, la de rotura, y la

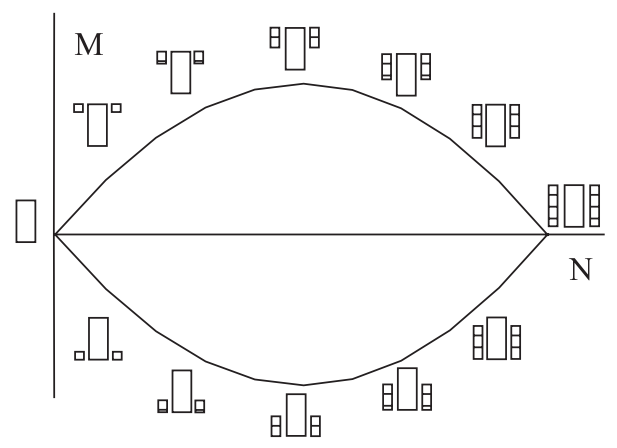

2. Superficie de fluencia en junta de arco de fábrica.

3. Superficie límite y criterios de seguridad en una junta de arco sin resistencia a tracción.

4. Corrección de una situación insegura dependiente del criterio empleado.
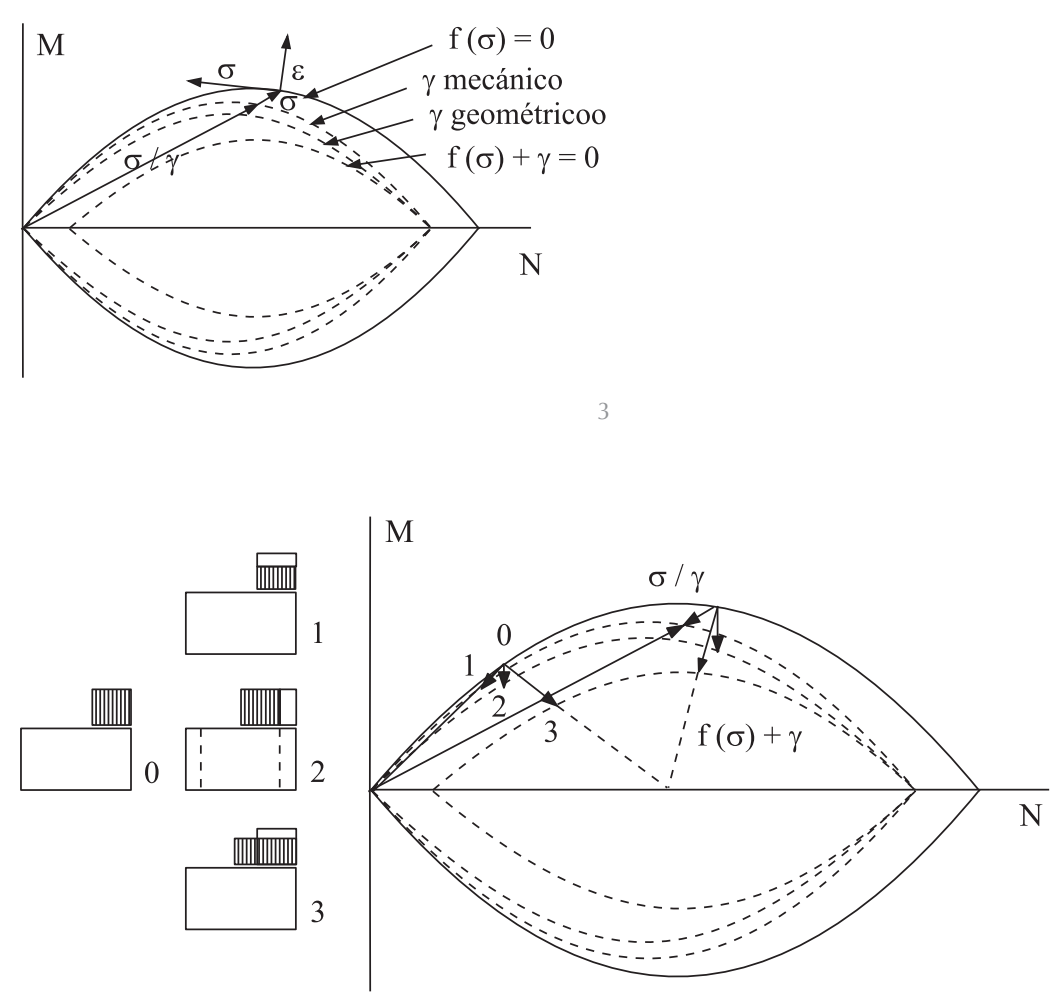

de comprobación empleada para el cálculo, versión homóloga y reducida en tamaño de aquélla, siendo dicho centro el punto fijo de la homología. Si dicho punto de carga o esfuerzo nulo no es el centro de la superficie de rotura, debemos corregir el criterio de comprobación de seguridad; las líneas siguientes aportan una línea sencilla para hacerlo.

Sea $\sigma$ un estado de carga - o de esfuerzoseguro, y sea $f(\sigma) \leq 0$ la superficie de rotura correspondiente a las variantes posibles para dicho estado cuyo centro - o posición de máximo alejamiento a dicha superficieesté localizado en $\sigma_{0}$. El estado $\sigma$ correspondería a un estado de rotura en el caso de una reducción de la superficie a una homóloga menor, y la relación entre las dimensiones lineales de dichas superficies puede dar la medida del margen de seguridad correspondiente al estado $\sigma$. 
5. Criterio robusto para medir el margen de seguridad.

${ }^{3}$ Tanto en esta expresión como en las sucesivas, se hace uso de operaciones vectoriales, de modo que las sumas y restas son de vectores, el punto representa producto escalar, etc...

${ }^{4}$ Efectivamente, si se da dicha coincidencia, $\sigma_{0}$ será el vector nulo, de modo que el coeficiente resultante, $\gamma=d / g \cdot \sigma$, cociente entre proyecciones sobre la ortogonal al plano, coincide por Tales con el coeficiente de seguridad mecánico habitual, cociente entre los módulos de los dos vectores colineales que representan uno, la Ilegada al plano de rotura, y otro, el estado de esfuerzos considerado.
El gradiente a la superficie en el punto de rotura más próximo al punto considerado define un vector $g$ ortogonal al plano tangente a ésta en dicha posición de rotura. Viendo la Figura 5, podemos considerar como margen de seguridad, análogo al coeficiente de seguridad habitual para las cargas, al cociente entre las proyecciones sobre dicha dirección de los dos vectores siguientes:

- El vector que representa al de los esfuerzos, pero medido desde el punto de máximo alejamiento a la superficie de rotura, más su distancia hasta el punto de rotura $\sigma-\sigma_{0}+\alpha g$;

- El vector de carga mismo, medido desde el mismo origen: $\sigma-\sigma_{0}$.

Es decir, el margen de seguridad se medirá por el cociente entre los segmentos $A C$ y $A B$ de la figura.

Nótese que si en la Figura 5 se consideran puntos arbitrarios, por ejemplo porque el procedimiento de análisis empleado comparase el estado de esfuerzos con todos los planos que aproximan la superficie, tendremos que según se aleja el punto $B$ que representa la proyección de los esfuerzos considerados del que representa la proyección de los esfuerzos de rotura $C$, el margen de seguridad crecerá hasta hacerse infinito cuando $\mathrm{B}$ coincida con $\mathrm{A}$, e incluso cambiará de signo si pasa a estar situado en la proyección más allá de $A$, es decir, si el centro de la figura quedase más cerca del plano de rotura considerado que el punto cuya distancia se comprueba.

La distancia entre el estado seguro y el de rotura próximo se puede definir con el vector, por ser proporcional al gradiente señalado, y el margen de seguridad definido más arriba puede medirse, por tanto con $^{3}$

$$
\gamma=\frac{\boldsymbol{g} \cdot\left(\boldsymbol{\sigma}-\boldsymbol{\sigma}_{0}+\alpha \boldsymbol{g}\right)}{\boldsymbol{g} \cdot\left(\boldsymbol{\sigma}-\boldsymbol{\sigma}_{0}\right)}
$$

Ahora bien, si consideramos el plano tangente como una aproximación de la superficie de rotura en el entorno considerado, la comprobación tendrá como forma general para cada uno de tales planos la expresión $g \cdot \sigma-d \leq 0$ expresión en la que si $g$ es unitario representará el versor del plano, y la comprobación mostrará que la proyección del vector que representa el estado considerado seguro sobre dicho versor debe ser menor que la distancia del origen - esfuerzo o carga nulos- a dicho plano. Nótese que la ecuación del plano considerado es precisamente la anterior particularizada para el caso de igualdad: $g \cdot \sigma-d=0$. Para este plano, el punto de rotura más próximo al del estado analizado se representa con $(\sigma+\alpha g)$

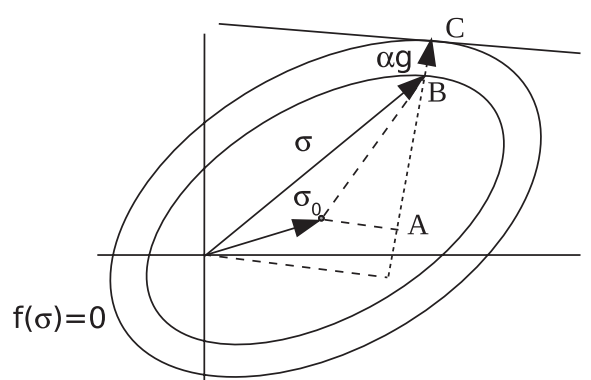

y para dicho punto de rotura se obtendría la igualdad:

$$
\boldsymbol{g} \cdot(\boldsymbol{\sigma}+\alpha \boldsymbol{g})-d=0
$$

expresión en la que podemos determinar fácilmente:

$$
\alpha=\frac{d-\boldsymbol{g} \cdot \boldsymbol{\sigma}}{\boldsymbol{g} \cdot \boldsymbol{g}}
$$

Resultará que:

- si $\alpha>0$, el estado es interior a la superficie, y por tanto seguro - con grado de seguridad a determinar-

- si $\alpha=0$, el estado es de rotura

- si $\alpha<0$, el estado es exterior a la superficie de rotura y por tanto imposible de alcanzar.

El margen de seguridad para el primero de los casos anteriores, siguiendo la ecuación descrita más arriba será:

$\gamma=\frac{\boldsymbol{g} \cdot(\boldsymbol{\sigma}+\alpha \boldsymbol{g})-\boldsymbol{g} \cdot \boldsymbol{\sigma}_{0}}{\boldsymbol{g} \cdot\left(\boldsymbol{\sigma}-\boldsymbol{\sigma}_{0}\right)}=\frac{d-\boldsymbol{g} \cdot \boldsymbol{\sigma}_{0}}{\boldsymbol{g} \cdot\left(\boldsymbol{\sigma}-\boldsymbol{\sigma}_{0}\right)}[2]$

De este modo, si podemos determinar los gradientes a la superficie - los planos tangentes a ésta en las regiones de interés-y las posiciones de tales planos tangentes de mayor cercanía a los estados a comprobar, la determinación del grado de seguridad es extremadamente sencilla, no presenta los inconvenientes derivados de la mayor o menor proximidad del punto de carga nula a la superficie límite, y finalmente es consistente con los modelos al uso en los casos en que el punto de carga nula y el centro de la superficie límite coinciden ${ }^{4}$.

\section{EJEMPLOS}

Como aplicación rápida del anterior criterio, procedemos al análisis de la resistencia en compresión excéntrica para secciones doblemente simétricas. Consideraremos tanto los casos en que exista resistencia en tracción - que consideraremos igual a la de compresión como es el caso de los metales- o el caso en que ésta sea despreciable. Para referirnos a cada uno de los 
casos, los denominaremos respectivamente metales y fábricas.

Para la simplificación que vamos a proponer supondremos además, como es habitual, que la superficie de fluencia para la combinación de esfuerzos de colapso por compresión excéntrica para cualquiera de los ejes es convexa, por lo que las superficies formadas por poliedros inscritos en dicha superficie están del lado de la seguridad.

Para determinar uno de los poliedros posibles, consideramos varias condiciones de colapso, de acuerdo con los seis criterios de la Figura 6, en la que pueden considerarse los dos sombreados como estados de tensión opuestos para los dos casos estudiados: en el caso de haber resistencia en tracción se tratará de estados de compresión o de tracción, según el sombreado, y en el caso de no haber resistencia en tracción, se tratará de una región comprimida y la otra sin tensión. Para cada criterio pueden determinarse las áreas resultantes y los momentos estáticos de dichas áreas con relación al centro de gravedad, que representarán adecuadamente las resistencias axiales, o los momentos resistentes, si multiplicamos dichas constantes estáticas por la resistencia unitaria del material empleado.

Por ejemplo, si de acuerdo con los criterios de la figura llamamos $A$ al área de la sección, y $E_{x}, E_{y}$ a los momentos estáticos de media sección respecto del centro de gravedad, tendremos que para el caso de materiales metálicos plásticos, y en la nomenclatura habitual en los Eurocódigos, las constantes estáticas relevantes serían el área $A, y$ los módulos plásticos $W_{p l, x}=$ $2 E_{x}$ y $W_{p l y}=2 E_{y^{\prime}}$ y tendríamos como límites de resistencia para los máximos esfuerzos positivos en cada uno de los los tres ejes independientes necesarios para representar los estados de flexocompresión o flexotracción:

$N_{R}=A f_{y} ; M_{x, R}=W_{p l, x} f_{y} ; M_{y, R}=W_{p l, y} f_{y}$

Hay que señalar que en la figura hay dos estados para los que la línea de paso de un estado de tensiones al opuesto es quebrada. Se trata de una simplificación del lado de la seguridad y por lo tanto admisible dado que, como puede verse en la Figura 7 , establecer una línea neutra recta que separe las dos regiones manteniendo los valores de las áreas sombreadas supone siempre el alejamiento del centro de gravedad de la región respecto de la posición que ocupa con la forma quebrada lo que incrementa obviamente los correspondientes momentos estáticos.
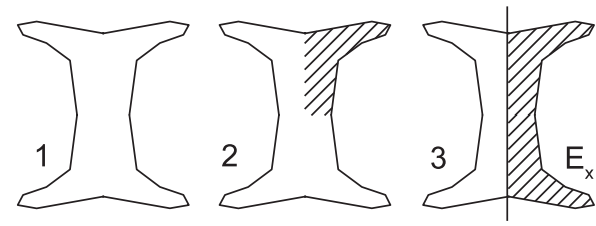

6. Vértices para el poliedro de rotura.
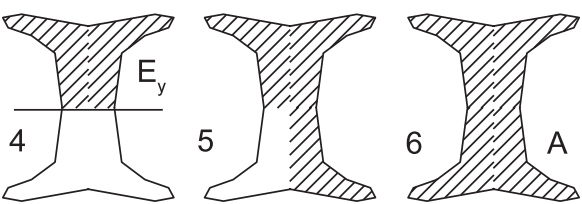

7. Línea neutra recta.

8. Poliedros de rotura

Tendremos, pues, que los valores estáticos representativos de las seis condiciones de rotura representadas en la figura 6 son, representados en formato vectorial, los siguientes:

\begin{tabular}{|c|c|c|}
\hline caso & metales & fábricas \\
\hline 1 & {$\left[\begin{array}{lll}-A & 0 & 0\end{array}\right]$} & {$\left[\begin{array}{lll}0 & 0 & 0\end{array}\right]$} \\
\hline 2 & {$\left[\begin{array}{lll}-\frac{1}{2} A & \frac{1}{2} W_{p l, x} & \frac{1}{2} W_{p l, y}\end{array}\right]$} & {$\left[\begin{array}{lll}\frac{1}{4} A & \frac{1}{4} W_{p l, x} & \frac{1}{4} W_{p l, y}\end{array}\right]$} \\
\hline 3 & {$\left[\begin{array}{lll}0 & W_{p l, x} & 0\end{array}\right]$} & {$\left[\begin{array}{lll}\frac{1}{2} A & \frac{1}{2} W_{p l, x} & 0\end{array}\right]$} \\
\hline 4 & {$\left[\begin{array}{lll}0 & 0 & W_{p l, y}\end{array}\right]$} & {$\left[\begin{array}{lll}\frac{1}{2} A & 0 & \frac{1}{2} W_{p l, y}\end{array}\right]$} \\
\hline 5 & {$\left[\begin{array}{lll}\frac{1}{2} A & \frac{1}{2} W_{p l, x} & \frac{1}{2} W_{p l, y}\end{array}\right]$} & {$\left[\begin{array}{lll}\frac{3}{4} A & \frac{1}{4} W_{p l, x} & \frac{1}{4} W_{p l, y}\end{array}\right]$} \\
\hline 6 & {$\left[\begin{array}{lll}A & 0 & 0\end{array}\right]$} & {$\left[\begin{array}{ll}A & 0\end{array}\right.$} \\
\hline
\end{tabular}

Estos valores definen, por tanto, los vértices de los poliedros de rotura que estamos considerando y que podemos ver representados en la Figura 8, cuya escala corresponde a los adimensionales de comprobación siguientes, que obtenemos por referencia a las resistencias plásticas de secciones de las que hemos Ilamado metálicas:

$$
n=\frac{N_{E}}{N_{R}}=\frac{N_{E}}{A f_{y}}, m_{x}=\frac{M_{E, x}}{M_{R, x}}=\frac{M_{E, x}}{W_{p l, x} f_{y}}, m_{y}=\frac{M_{E, y}}{M_{R, y}}=\frac{M_{E, y}}{W_{p l, y} f_{y}} .
$$

Se están representando sólo las regiones positivas en los valores de los momentos; las negativas podrían obtenerse por simetría.

Vemos que en dicha figura, los centros de cada poliedro corresponden a las coordenadas $\sigma_{0, m}=\left(\begin{array}{lll}0 & 0 & 0\end{array}\right) ; \sigma_{0, f}=\left(\begin{array}{lll}0,5 & 0 & 0\end{array}\right)$. Podemos, pues, determinar las comprobaciones apli-
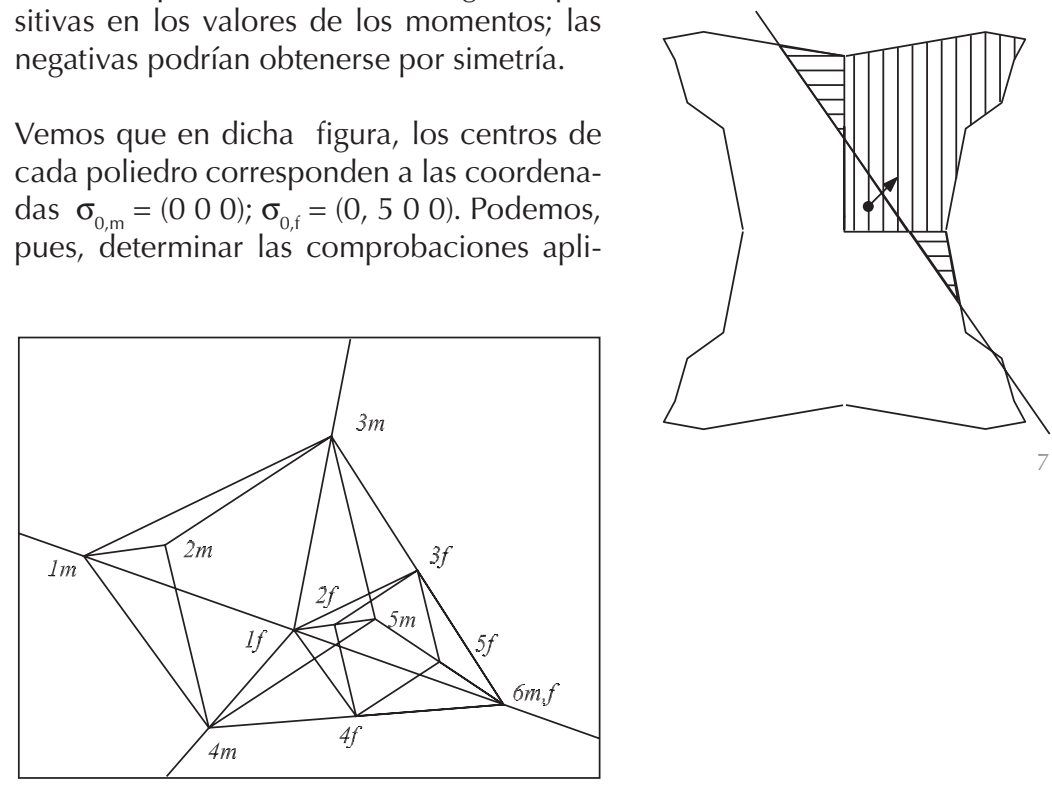
cando los criterios definidos en el apartado anterior.

Consideremos el caso de los metales. Tendremos las comprobaciones de la tabla siguiente, fáciles de deducir de la expresión que resulta para la condición de $\sigma_{0, \mathrm{~m}}=(0$ $00)$ :

$$
\gamma_{m, i}=\frac{d}{\boldsymbol{g} \cdot \boldsymbol{\sigma}}
$$

\begin{tabular}{|c|ccc}
\hline plano & vértices & ecuación del plano & comprobación \\
\hline \multirow{3}{*}{1} & {$\left[\begin{array}{ccc}1 & 0 & 0 \\
0 & 1 & 0 \\
0,5 & 0,5 & 0,5\end{array}\right]$} & $n+m_{x}=1$ & $\gamma_{m, 1}=\frac{1}{n+m_{x}}$ \\
3 & {$\left[\begin{array}{ccc}1 & 0 & 0 \\
0 & 0 & 1 \\
0,5 & 0,5 & 0,5\end{array}\right]$} & $n+m_{y}=1$ & $\gamma_{m, 2}=\frac{1}{n+m_{y}}$ \\
4 & {$\left[\begin{array}{ccc}70,5 & 0,5 & 0,5 \\
0 & 1 & 0 \\
0 & 0 & 1\end{array}\right]$} & $m_{x}+m_{y}=1$ & $\gamma_{m, 3}=\frac{1}{m_{x}+m_{y}}$ \\
5 & {$\left[\begin{array}{ccc}-0,5 & 0,5 & 0,5 \\
0 & 1 & 0 \\
-1 & 0 & 0\end{array}\right]$} & $-n+m_{x}=1$ & $\gamma_{m, 4}=\frac{1}{-n+m_{x}}$ \\
0 & {$\left[\begin{array}{ccc}-0,5 & 0,5 & 0,5 \\
0 & 0 & 1 \\
-1 & 0 & 0\end{array}\right]$} & $-n+m_{y}=1$ & $\gamma_{m, 5}=\frac{1}{-n+m_{y}}$ \\
\hline
\end{tabular}

Nótese que las comprobaciones numeradas 1 y 4 y las 2 y 5 , son en realidad las mismas salvo el signo en los esfuerzos axiales. Las comprobaciones precisas son las 1, 2 y 3 si consideramos sólo las comprobaciones que corresponden a valores positivos, o absolutos, en $n, m x, m y$, es decir, empleando sólo el triedro positivo del poliedro de rotura.

Véase que si hubiésemos ignorado los vértices numerados $2 m$ y $5 m$ del poliedro, de modo a aproximar la superficie en el triedro positivo sólo con un plano, la comprobación que resultaría ahora sería:

\begin{tabular}{|c|rrrr|}
\hline plano & \multicolumn{2}{|c|}{ vértices } & ecuación del plano & comprobación \\
\hline \multirow{2}{*}{0} & {$\left[\begin{array}{lll}1 & 0 & 0 \\
0 & 1 & 0 \\
0 & 0 & 1\end{array}\right]$} & $n+m_{x}+m_{y}=1$ & $\gamma_{m, 0}=\frac{1}{n+m_{x}+m_{y}}$ \\
\hline
\end{tabular}

Dicha comprobación es la habitual en los códigos, y como puede verse es innecesariamente conservadora, pudiendo ser sustituída con generalidad por el conjunto de las tres primeras de la tabla precedente que, como bien se ve, sólo contrastan la interacción del esfuerzo axial o los momentos flectores para cada uno de los dos planos de flexión, considerados de dos en dos.

Si consideramos ahora el caso de las secciones de fábrica, sin resistencia a la tracción, para la condición de $\sigma_{0, f}=\left(\begin{array}{llll}0 & 0 & 0\end{array}\right)$ tendremos las comprobaciones: $\gamma_{f, i}=\frac{d-\boldsymbol{g} \cdot \boldsymbol{\sigma}_{0, f}}{\boldsymbol{g} \cdot\left(\boldsymbol{\sigma}-\boldsymbol{\sigma}_{0, f}\right)}=\frac{d-0,5 a_{g}}{\boldsymbol{g} \cdot \boldsymbol{\sigma}-0,5 a_{g}}$

donde $\mathrm{a}_{\mathrm{g}}$ es el coeficiente del primer término de la ecuación del plano $g \cdot \sigma-d=$ 0 , en la que $g=\left(\mathrm{a}_{g} \mathrm{~b}_{g} \mathrm{c}_{\mathrm{g}}\right)^{(*)}$, aunque resulta preferible representar las comprobaciones en la forma ${ }^{(* *)}$

Hay que hacer notar que en las comprobaciones aplicadas a las secciones que hemos denominado fábricas, estamos empleando como referencia para las resistencias las de los metales, lo que no supone diferencia en el caso del límite para esfuerzos axiales $N_{R^{\prime}}$ pero sí para los máximos flectores admisibles, que es doble si hay resistencia en tracción. Es decir, estamos determinando $m_{x}=M_{E, X} / M_{R, X}$ empleando para el valor de $M_{R \times X}$ el obtenido a partir del módulo plástico, considerando tracciones. Hemos hecho eso para facilitar la comparación entre ambos casos, pero sería razonable que las máximas resistencias se determinen a partir de las cualidades propias del problema considerado. Si estamos hablando de fábricas sin considerar el recurso a las tracciones podemos rehacer el razonamiento completo empleando ahora como vértices del poliedro los puntos:

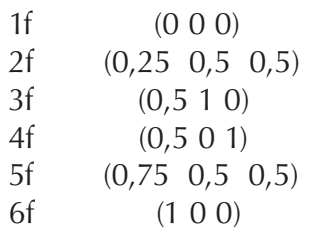

y con ello determinar las comprobaciones resultantes. Es fácil ver, sin embargo, que en el caso que estamos analizando de secciones doblemente simétricas la máxima resistencia axial es idéntica, mientras que los máximos flectores admitidos son mitad de los de las secciones metálicas, por lo que si referimos los correspondientes adimensionales a dicho valor mitad, las comprobaciones que ya hemos obtenido se transformarán $\mathrm{en}^{(* *)}$. Como ya se ha dicho, el margen - o coeficiente- de seguridad de la sección para los esfuerzos considerados sería el positivo de menor valor de entre los obtenidos, recordando que los valores negativos tienen como significado el hecho de que el plano que se emplea para la comprobación estaría más lejos del punto que representa los esfuerzos que se comprueban que el centro del poliedro.

Podemos comparar ahora el resultado que nuestro criterio de comprobación aporta para determinar la seguridad en la altura de un prisma rígido apoyado en su base cuadrada, comparando con los criterios habi- 


\begin{tabular}{|c|c|c|c|c|c|}
\hline plano & \multicolumn{3}{|c|}{ vértices } & ecuación del plano & comprobación \\
\hline \multirow{3}{*}{1} & 1 & 0 & 0 & \multirow{3}{*}{$n+m_{x}=1$} & \multirow{3}{*}{$\gamma_{f, 1}=\frac{0,5}{n+m_{x}-0,5}$} \\
\hline & 0,5 & 0,5 & 0 & & \\
\hline & 0,75 & 0,25 & 0,25 & & \\
\hline \multirow{3}{*}{2} & 1 & 0 & 0 & \multirow{3}{*}{$n+m_{y}=1$} & \multirow{3}{*}{$\gamma_{f, 2}=\frac{0,5}{n+m_{y}-0,5}$} \\
\hline & 0,5 & 0 & 0,5 & & \\
\hline & 0,75 & 0,25 & $0,25]$ & & \\
\hline \multirow{3}{*}{3} & $\mp 0,75$ & 0,25 & 0,25 & \multirow{3}{*}{$m_{x}+m_{y}=0,5$} & \multirow{3}{*}{$\gamma_{f, 3}=\frac{0,5}{m_{x}+m_{y}}$} \\
\hline & 0,5 & 0,5 & 0 & & \\
\hline & 0,5 & 0 & 0,5 & & \\
\hline \multirow[b]{2}{*}{4} & {$[0,25$} & 0,25 & $0,25]$ & \multirow[b]{2}{*}{$-n+m_{x}=0$} & \multirow{2}{*}{$\gamma_{f, 4}=\frac{0,5}{-n+m_{x}+0,5}$} \\
\hline & $\begin{array}{l}0 \\
0\end{array}$ & $\begin{array}{c}0,5 \\
0\end{array}$ & $\begin{array}{l}0 \\
0\end{array}$ & & \\
\hline \multirow{3}{*}{5} & $=0,25$ & 0,25 & $0,25]$ & \multirow{3}{*}{$-n+m_{y}=0$} & \multirow{3}{*}{$\gamma_{f, 5}=\frac{0,5}{-n+m_{y}+0,5}$} \\
\hline & 0 & 0 & 0,5 & & \\
\hline & 0 & 0 & 0 & & \\
\hline
\end{tabular}

$(* *)$

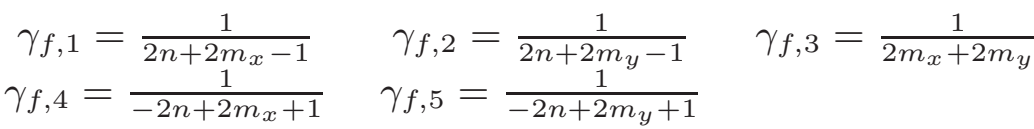

$(* * *)$

$$
\begin{aligned}
\gamma_{f, 1} & =\frac{1}{2 n+m_{x}-1} & \gamma_{f, 2} & =\frac{1}{2 n+m_{y}-1} \\
\gamma_{f, 4} & =\frac{1}{-2 n+m_{x}+1} & \gamma_{f, 5} & =\frac{1}{-2 n+m_{y}+1}
\end{aligned}
$$

donde ahora

$n=\frac{N_{E}}{N_{R}}=\frac{N_{E}}{A f_{y}}, m_{x}=\frac{M_{E, x}}{M_{R, x}}=\frac{M_{E, x}}{E_{x} f_{y}}, m_{y}=\frac{M_{E, y}}{M_{R, y}}=\frac{M_{E, y}}{E_{y} f_{y}}$.

tuales. Sea el prisma de base cuadrada de lado a y altura $h$ con peso específico $\rho$. Si consideramos como efecto de perturbación para su equilibrio una fuerza horizontal $F$, situada en lo alto del prisma, tendremos como resultantes de esfuerzos los tres valores $\left(a^{2} h \rho\right.$ Fh 0$)$.

Consideremos ahora varios criterios de seguridad. El primero, clásico para el análisis de la estabilidad frente al vuelco, comparará los momentos estabilizante y desestabilizante suponiendo el vuelco en torno a la arista exterior.

$$
1 \leq \gamma_{1}=\frac{a^{3} \rho \frac{h}{2}}{F h}=\frac{\rho a^{3}}{2 F} .
$$

El segundo corresponderá a la comprobación de resistencia. Si suponemos que la base es capaz de soportar una tensión máxima $f$, podemos usar ahora la comprobación clásica que combina linealmente las pérdidas de resistencia derivadas de tener que soportar simultáneamente axil y momento. Dicha expresión se usa sin dificul- tad en el caso de secciones con resistencia en tracción -y es la habitual en comprobaciones plásticas en metales tal como las define, por ejemplo, el Eurocódigo 3- pero no es evidente su forma de empleo cuando los momentos se resisten sólo mediante las regiones comprimidas excéntricamente.

Usaremos, pese a todo, el área y el momento estático de media sección comprimida como referencias para los mayores valores de resistencia que puedan alcanzarse, de modo que:

$n=\frac{\rho a^{2} h}{a^{2} f}=\frac{\rho h}{f} ; m_{x}=\frac{F h}{\frac{a^{3}}{8} f}=8 \frac{F h}{a^{3} f}=4 \frac{2 F \rho h}{\rho a^{3} f}=\frac{4 n}{\gamma_{1}}$

expresión en la que hemos usado el valor obtenido para $\gamma_{1}$ en la expresión precedente. Si establecemos ahora la forma de interacción lineal de forma ingenua tendríamos como criterio de seguridad:

$$
1 \leq \gamma_{2}=\frac{1}{n+m_{x}}=\frac{1}{n\left(1+\frac{4}{\gamma_{1}}\right)}
$$


En contraste empleamos ahora el criterio que acabamos de establecer, para el apoyo sin resistencia en tracción, y con tensiones de compresión limitadas al valor $f$, tendremos como tercer criterio de resistencia el siguiente, considerando sólo los dos planos de interacción entre el esfuerzo axial y el momento en una dirección:

$1 \leq \gamma_{3}=\min \left(\frac{1}{2 n+m_{x}-1}, \frac{1}{-2 n+m_{x}+1}\right)$

o lo que es lo mismo,

$$
\begin{aligned}
& 1 \leq \gamma_{3} \leq \frac{1}{n\left(2+\frac{4}{\gamma_{1}}-\frac{1}{n}\right)} \\
& 1 \leq \gamma_{3} \leq \frac{1}{n\left(-2+\frac{4}{\gamma_{1}}+\frac{1}{n}\right)}
\end{aligned}
$$

Podemos finalmente comparar las prescripciones de dichos criterios de seguridad. Los requisitos establecidos por las versiones clásicas -comprobación de vuelco, y resistencia combinada a hundimiento y vuelco-son:

$$
\begin{gathered}
\gamma_{1} \geq 1 \\
n \leq \frac{1}{1+\frac{4}{\gamma_{1}}}
\end{gathered}
$$

El tercer criterio aquí propuesto aporta las condiciones siguientes:

$$
\begin{gathered}
n \leq \frac{1}{2+\frac{4}{\gamma_{1}}-\frac{1}{n}} ; \frac{1}{n} \geq 2+\frac{4}{\gamma_{1}}-\frac{1}{n} ; \frac{1}{n} \geq 1+\frac{2}{\gamma_{1}} ; n \leq \frac{1}{1+\frac{2}{\gamma_{1}}} \\
n \leq \frac{1}{-2+\frac{4}{\gamma_{1}}+\frac{1}{n}} ; \frac{1}{n} \geq-2+\frac{4}{\gamma_{1}}+\frac{1}{n} ; 2 \geq \frac{4}{\gamma_{1}} ; \gamma_{1} \geq 2
\end{gathered}
$$

Como se ve, el criterio que acabamos de establecer aporta por sí solo dos expresiones semejantes a las ya obtenidas mediante los dos criterios anteriores. Se trata de expresiones cuya confiabilidad es mayor.

Si empezamos por la segunda de ellas, la diferencia contra la clásica expresión de estabilidad contra el vuelco deriva de que, al no poderse considerar el apoyo en la arista exterior como si fuese infinitamente rígido y resistente, el grado de seguridad que aporta la expresión clásica es diferente, en contra de la seguridad. La expresión alternativa que se presenta aquí refleja que el mayor mo-

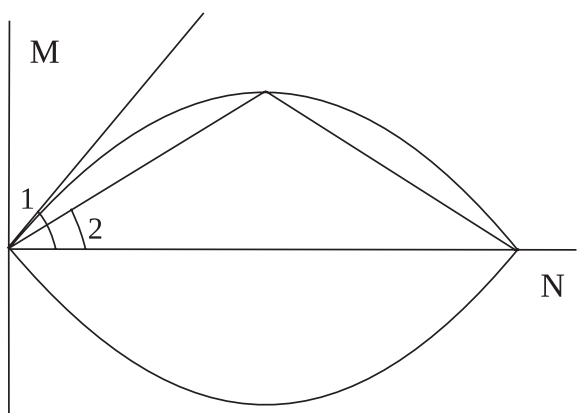

mento estabilizante que cabe obtener para una resistencia dada en el terreno de apoyo sería el de media sección del apoyo completamente comprimida, $y$, por tanto, con brazo mitad respecto al considerado en la comprobación clásica. Para otras áreas comprimidas, tanto si son mayores como si son menores, el momento estabilizante resultará siempre menor que dicho máximo. Al aproximar el coeficiente de seguridad mediante una sola expresión para todos los casos resulta un factor de 2 en menor eficacia para el caso límite en que no hay casi carga, ni por lo tanto momento de vuelco. Una visión en detalle de la superficie de fluencia en las proximidades del punto de carga nula comparada con la superficie aproximada por los planos del poliedro generado a partir de los casos de rotura considerados - los situados entre la separación por tracción y el vuelco con hundimiento de media sección, ver figura 9- aclara la diferencia entre ambos valores: la forma exacta corresponde a la llegada a dicho punto de la rama de una parábola, que pasa por los puntos que representan ambos casos, frente a una recta para la versión propuesta aquí. La pendiente de la rama de la parábola en el origen es doble a la pendiente de la recta. La expresión clásica frente a vuelco supone usar como aproximación a dicha rama parabólica la recta tangente a ella en el origen, lo que está en contra de seguridad. La expresión aquí propuesta usa, en vez, la recta secante, segura, aunque más conservadora para los casos de casi ni carga ni vuelco.

La primera de las dos comprobaciones obtenidas con el criterio aquí propuesto establece un límite a la carga que puede suponer el peso del bloque en relación con la carga centrada que puede soportar el apoyo. La expresión para ese límite es de mismo tipo al obtenido mediante un razonamiento clásico y un diagrama de interacción lineal, y permite valores superiores a dicha relación, si bien ahora, dado que no hay confusión posible en cómo establecer las resistencias máximas en carga y en carga excéntrica, la expresión resulta completamente satisfactoria. El error en la expresión inicial deriva precisamente de haber considerado normal y momento máximos como independientes al construir la recta de interacción, cuando en realidad el momento máximo está ligado a un normal mitad del máximo.

\section{CONCLUSIONES}

Se ha propuesto un criterio de seguridad que considera condiciones de rotura no necesariamente isótropas, y no necesariamente centradas respecto del punto de carga o esfuerzo nulo. 
Se ha comprobado que dicho criterio resulta consistente con los habituales en los casos en que las condiciones de colapso son simétricas respecto de la situación de carga o esfuerzo nulo.

Se ha comprobado que dicho criterio puede aplicarse con eficacia y gran facilidad tanto al análisis de la resistencia de secciones de materiales perfectamente plásticos y con simetría en el comportamiento en tracciones y compresiones, como al análisis de la resistencia de secciones de materiales sin resistencia a la tracción.

En el caso de materiales plásticos y simétricos se ha comprobado que la aplicación de dicho criterio - que resulta en este caso idéntico al habitual - a secciones doblemente simétricas demuestra que en la comprobación de estados de flexocompresión esviada basta comprobar la resistencia para la interacción separada del esfuerzo axial con el momento sea en una o en otra de las direcciones, y la interacción de ambos momentos conjuntamente pero sin interacción del esfuerzo axial, es decir, considerando sólo todas las interacciones entre dichos esfuerzos tomados de dos en dos.

Se ha comprobado, finalmente, que dicho criterio permite unificar en un solo modelo criterios de comprobación considerados hasta ahora independientes, como es el caso de las condiciones de seguridad al vuelco de un prisma apoyado en una base de resistencia dada.

\section{ANEJO 1: BREVES CONSIDERACIONES TÉCNICAS}

La unificación entre los criterios históricamente diferenciados de estabilidad y de resistencia puede interpretarse con facilidad si obtenemos las comprobaciones de estabilidad como límite de series de comprobaciones de resistencia en las que la resistencia a tracción de las fábricas tiende a cero.

Puede asimismo justificarse el empleo de una función $F(\Sigma)$ para expresar la condición de rotura en casos en que $\Sigma$ represente valores agregados representativos de un comportamiento de sección —o pieza o estructura-. Para ello bastará

- que el material empleado tenga definida una función de plastificación $f(\sigma)$ válida para las tensiones de punto $\sigma$-cuyas correspondientes deformaciones se denotan con $\varepsilon$ - función para la que sea correcta la condición de ortogonalidad en la situación límite:

$$
\frac{\partial f}{\partial \sigma}=\lambda \varepsilon
$$

- que la caracterización del equilibrio para el comportamiento agregado pueda hacerse a través de parámetros de esfuerzo - $\mathrm{O}$ incluso de carga - $\Sigma$ determinables a partir de los $\sigma$ mediante expresiones lineales del tipo $\Sigma=\int_{\Omega} L(\sigma) d \Omega$, siendo $L(\cdot)$ un operador lineal, tal vez diferencial, sobre $\sigma$.

- que la caracterización cinemática del comportamiento agregado se haga mediante parámetros cinemáticos $E$ tales que las deformaciones $\varepsilon$ puedan deducirse de ellos a través de la expresión $\sigma=L^{T}(E)$ donde $L^{T}(\cdot)$ es el operador adjunto de $L(\cdot)$, es decir:

$$
\int_{\Omega} a^{T} \cdot L(b) d \Omega=\int_{\Omega} L^{T}(a) \cdot b d \Omega
$$

- que la función $F(\Sigma)$ pueda construirse mediante alguna expresión del tipo: $F(\Sigma)=\int_{\Omega} A(f(\sigma)) d \Omega$, siendo $A$ constante en el dominio, para lo que debe definirse alguna forma para la relación $\Sigma(\sigma)$-lo que equivaldría a algún tipo de pseudoinversa, pero no necesariamente la inversa matemática, de la relación: $\sigma(\Sigma)=\int_{\Omega} L(\sigma) d \Omega$, ya señalada antes-

La segunda y tercera condiciones aseguran que el cambio de representación de cualesquiera condiciones estáticas y cinemáticas $\sigma, \dot{\varepsilon}$ a sus transformadas $\Sigma, \dot{E}$ se producen respetando la invariancia en los trabajos según la expresión: $\int_{\Omega} \dot{\varepsilon}^{T} \cdot \sigma d \Omega=\dot{E}^{T} \cdot \Sigma$.

Todas ellas aseguran para $F$ el cumplimiento de la condición básica de ortogonalidad en la situación de flujo plástico, expresada por:

$$
\frac{\partial F}{\partial \Sigma}=\Lambda E
$$

Un ejemplo conocido de estas condiciones es el de una sección de fábrica plastificando por tensiones normales y sin capacidad en tracción para alguna combinación de esfuerzos normales $N=\int_{A} \sigma_{x}(z) d A$ y momentos flectores $M=\int_{A} z \sigma_{x}(z) d A$. En este caso tenemos:

$$
\begin{gathered}
\sigma=\sigma_{x}(z) ; \Sigma=\left[\begin{array}{l}
N \\
M
\end{array}\right] ; L=\left[\begin{array}{l}
1 \\
x
\end{array}\right] ; \\
E=\left[\begin{array}{l}
\epsilon \\
c
\end{array}\right] \operatorname{con} \varepsilon=\epsilon+x c=\left[\begin{array}{ll}
1 & x
\end{array}\right]\left[\begin{array}{l}
\epsilon \\
c
\end{array}\right]
\end{gathered}
$$

$y$, definiendo $f(\sigma)$ como el negativo de la menor diferencia entre la tensión atribuída al punto y la máxima en compresión o la tensión nula en tracción, estableciendo $F(\Sigma)$ mediante la integral simple de $f(\sigma)$ en todos los puntos de la sección, e interpolando, para obtener una descripción definida de $\Sigma(\sigma)$, las condiciones de solicitación que no alcancen la rotura como intermedios entre el estado de compresión centrada mitad de la máxima y el estado de rotura en la dirección del alejamiento de los esfuerzos existentes. 
La última cuestión a considerar responde a la métrica del espacio en que se representa la función de rotura: es evidente que en espacios cuyos diferentes ejes corresponden a agregados, las magnitudes o escalas de cada dimensión pueden no ser comparables en absoluto, lo que hace que medir en direcciones oblicuas a dichos ejes pueda carecer totalmente de sentido. En estos espacios, las unidades elegidas deben responder a criterios de incertidumbre, es decir, deben asegurar que una esfera de radio dado represente en cada dirección una incertidumbre similar en los valores de las magnitudes representadas. Esto nos remite a la necesidad de validar las escalas sobre las que se aplica el procedimiento descrito mediante criterios probabilísticos que aseguren dispersiones similares para todas las magnitudes.

En defecto de mayor precisión, la vía empleada aquí responde al procedimiento habitual de formular los valores de estado para cada eje en proporción al valor de la resistencia máxima en dicho eje sin interacción del resto. Para considerar avances en esta dirección puede resultar útil (4).

\section{REFERENCIAS}

(1) D'Ayala, D.F. and Tomasoni, E.: "The structural behaviour of masonry vaults: Limit state analysis with finite friction". Structural Analysis of Historic Construction, pages 3 - 19, 2008.

(2) Augusti, G.; Baratta, A. and Casciati, F.: "Probabilistic Methods in Structural Engineering". Chappman and Hall, London, New York, 1984.

(3) Livesley, R.K.: "Limit analysis of structures formed from rigid blocks". International Journal for Numerical Methods in Engineering, 12:1853-1871, 1978.

(4) Marti, K. "Limit load and shakedown analysis of plastic structures under stochastic uncertainty". Computer Methods in Applied Mechanics and Engineering, 198-1:42 - 51, 2008.

(5) Nielsen, M.P. "Limit analysis and concrete plasticity". CRC Press, Boca Raton, Florida, 1998. $2^{a}$ edición. 